AperTO - Archivio Istituzionale Open Access dell'Università di Torino

Thymidylate synthase is functionally associated with ZEB1 and contributes to the epithelial-tomesenchymal transition of cancer cells

This is a pre print version of the following article:

Original Citation:

Availability:

This version is available http://hdl.handle.net/2318/1671514

since 2018-07-30T12:54:19Z

Published version:

DOI:10.1002/path.4897

Terms of use:

Open Access

Anyone can freely access the full text of works made available as "Open Access". Works made available under a Creative Commons license can be used according to the terms and conditions of said license. Use of all other works requires consent of the right holder (author or publisher) if not exempted from copyright protection by the applicable law. 


\section{Thymidylate synthase is functionally associated with ZEB1 and contributes to the epithelial-to- mesenchymal transition of cancer cells}

Aarif Siddiqui', Maria Eleni Vazakidou', Annemarie Schwab ${ }^{1}$, Francesca Napoli, Cristina Fernandez-Molina ${ }^{1}$, Ida Rapa ${ }^{2}$, Marc P. Stemmler ${ }^{3}$, Marco Volante ${ }^{2}$, Thomas Brabletz $z^{3}$ and Paolo Ceppi ${ }^{1}$.

1. Junior Research Group 1, Interdisciplinary Center for Clinical Research, Friedrich-Alexander-University Erlangen-Nürnberg (FAU), Erlangen, Germany.

2. Pathology Unit, San Luigi Hospital, University of Turin, Turin, Italy;

3. Experimental Medicine I, Friedrich-Alexander-University Erlangen-Nürnberg (FAU), Erlangen, Germany.

Correspondence to: Dr. Paolo Ceppi, Junior Research Group 1, Interdisciplinary Center for Clinical Research, Friedrich-Alexander-University Erlangen-Nürnberg (FAU), Nikolaus-Fiebiger-Zentrum, Room 0.032, Glückstrasse 6, D-91054 Erlangen, Germany. Phone:+49 (0)91318539300. Fax:+49 (0)91318536386.

The authors declare no conflicts of interests.

Running title: Thymidylate synthase in cancer EMT

Word count: 3954 words

Main Figures: 6 


\section{ABSTRACT}

Thymidylate synthase (TS) is a fundamental enzyme of nucleotide metabolism and one of the oldest anti-cancer targets. Beginning from the analysis of gene array data from the $\mathrm{NCl}-60$ panel of cancer cell lines, we identified a significant correlation at both gene and protein level between TS and the markers of epithelial-to-mesenchymal transition (EMT), a developmental process that allows cancer cells to acquire features of aggressiveness, like motility and chemoresistance. TS levels were found to be significantly augmented in mesenchymal-like compared to epithelial-like cancer cells, to be regulated by EMT induction, and to negatively correlate with micro-RNAs (miRNAs) usually expressed in epithelial-like cells and known to actively suppress EMT. Transfection of EMT-suppressing miRNAs reduced TS levels, and a specific role for miR-375 in targeting the TS 3 '-untranslated region was identified. A particularly relevant association was found between TS and the powerful EMT driver ZEB1, the shRNAmediated knockdown of which up-regulated miR-375 and reduced TS cellular levels. The TS-ZEB1 association was confirmed in clinical specimens from lung tumors and in a genetic mouse model of pancreatic cancer with ZEB1 deletion. Interestingly, TS itself appeared to have a regulatory role on EMT in cancer cells, as TS knockdown could directly reduce the EMT phenotype, migratory ability of cells, the expression of stem-like markers and chemoresistance. Taken together, these data indicate that the TS enzyme is functionally linked with EMT and cancer differentiation, with several potential translational implications.

\section{Keywords:}

- thymidylate synthase

- epithelial-to-mesenchymal transition

- ZEB1

- micro-RNAs

- solid tumors 


\section{INTRODUCTION}

Despite the advances made in recent years, for most types of solid tumors only a minority of selected patients benefit from molecularly targeted agents, while the majority (including relapsing patients) are treated with conventional cytotoxic chemotherapy [1]. There is therefore an urgent need for the development of novel drugs and strategies based on a better understanding of cancer biology and of the mechanisms of chemoresistance.

Thymidylate synthase (TS) is the enzyme catalyzing the conversion of deoxyuridine monophosphate (dUMP) to thymidine monophosphate (dTMP, thymidylate). Since this reaction provides the sole de novo pathway for thymidylate production, TS is essential for DNA synthesis and repair, and its absence blocks proliferation and causes cell death [2]. TS is greatly overexpressed in most cancers and associates with metastatic spread and reduced overall survival [3-6]. TS is known as a target of chemotherapy, being inhibited by the pyrimidine analogue 5 -fluorouracil (5-FU) or by folate analogues. Its overexpression in tumors represents a major mechanism of intrinsic chemoresistance and, as a result, conventional pharmacological inhibition shows limited efficacy $[7,8]$. Moreover, as an acquired resistance mechanism, TS expression levels are rapidly up-regulated following treatment with chemotherapeutic drugs, including agents that inhibit its activity $[9,10]$. Importantly, TS has also been shown to function as an oncogene, as stable TS transfection into murine fibroblasts induced a transformed phenotype, anchorage-independent growth and tumor formation in nude mice [11].

Epithelial-to-mesenchymal transition (EMT) is a developmental process used by cancer cells to acquire a more invasive phenotype [12]. This is accompanied by the loss of epithelial polarity (with the reduction of adhesion molecules, like E-cadherin) and the acquisition of mesenchymal markers like vimentin, $\mathrm{N}$-cadherin and others, and requires the involvement of specific reprogramming factors [13]. EMT has also been implicated as a mechanistic motor of cancer stem cells (CSCs) [14], which are undifferentiated cancer cells with stem-like features responsible for tumor heterogeneity and for some of the most lethal features of cancers [15,16]. The mechanisms behind EMT and CSCs 
are currently the object of several investigations, with great expectations in terms of therapeutic development.

A few enzymes of nucleotide metabolism and associated pathways have been recently functionally connected with EMT and the maintenance of a CSC phenotype $[17,18]$. We therefore aimed at determining a possible correlation between TS and EMT. By data mining, cell biology techniques and immunohistochemical staining of tumor samples, we identified here an unprecedented association, and described a regulatory axis that involves the EMT driver ZEB1 and relevant miRNAs.

\section{METHODS}

Cell lines. The hepatocellular carcinoma cell line HepG2 was cultured in EMEM (ATCC, Manassas, VA, USA). The colon carcinoma cell lines HCT116 and HT29 were cultured in McCoy's 5A medium (Lonza, Basel, Switzerland), 293T cells were cultured in DMEM (Sigma-Aldrich, St. Louis, MO, USA). KPC cells from the mouse model of pancreatic ductal adenocarcinoma were cultured in DMEM. All other cells were cultured in RPMI1640 (Sigma-Aldrich). All media were supplemented with 10\% fetal bovine serum, 1\% penicillin/streptomycin and 1\% L-glutamine (Sigma-Aldrich).

Reagents and antibodies. For Western blotting the antibody for TS (EPR4545) was from Abcam (Cambridge, UK); antibodies to E-cadherin (4A2), vimentin (D21H3) and $\beta$ actin (8H10D10) from Cell Signaling (Danvers, MA, USA); anti-ZEB1 (HPA027524) is from Sigma-Aldrich; anti-Oct-4 (9B7) and anti-nanog (PA1-097) from Thermo-Fisher (Waltham, MA USA). Secondary antibodies were from Southern Biotech (Birmingham, AL, USA). Bovine serum albumin is from Carl Roth (Karlsruhe, Germany). Blockinggrade milk is from BioRad (Hercules, California, USA). Puromycin and propidium iodide are from Sigma-Aldrich. 5-fluorouracil powder was reconstituted in DMSO (both from Sigma-Aldrich). Cisplatin was obtained from Santa Cruz Biotech (Dallas, TX, USA) and 
reconstituted in sterile water. Recombinant TGF- $\beta$ and StemXVivo EMT-inducing supplement are from R\&D Systems (Minneapolis, MN, USA).

Patients and samples. Formalin-fixed paraffin-embedded surgical specimens of tumor tissues of 61 NSCLC patients completely resected between 2005 and 2006 at the San Luigi Hospital were consecutively collected. The main characteristics of the patients are shown in Figure S8. None of the patients received pre-surgical chemotherapy or radiation therapy. All cases were reviewed and classified using anonymized samples. The use of retrospective solid tumor tissues for the immunohistochemical study was approved by the Research Ethics Committee of the San Luigi Hospital/University of Turin (approvals n.167/2015 and 204/2016).

Immunohistochemistry staining and scoring. Tissues were de-waxed, rehydrated and processed for staining. Antigen retrieval was performed in a PT-Link pretreatment system from Dako (Glostrup, Denmark) for 40 min at $98{ }^{\circ} \mathrm{C}$, using pH 9.0 for TS and ZEB1 antibodies and pH 6.0 for E-cadherin. After blocking endogenous peroxidase in $3 \%$ hydrogen peroxide and blocking non-specific sites with $1 \%$ BSA for $30 \mathrm{~min}$, the slides were incubated for $1 \mathrm{~h}$ at room temperature with primary antibodies: TS (EPR4545 from Abcam, 1:150 dilution), E-cadherin (36B5 from NeoMarkers/ThermoFisher, 1:30) and ZEB1 (HPA027524 from Sigma-Aldrich, 1:100). The sections were then incubated for $40 \mathrm{~min}$ with anti-rabbit (TS and ZEB1) or anti-mouse (E-cadherin) Dako REAL Envision-HRP. After washing, slides were incubated with DAB for 10 min and then counterstained with hematoxylin, dehydrated and mounted. Immunoreactivity was evaluated independently by two pathologists by $\mathrm{H}$-score, calculated as follows: $\mathrm{H}$ score $=\Sigma \mathrm{P}_{\mathrm{i}}(\mathrm{i}+1)$, where $\mathrm{i}$ represents the intensity of staining $(0-3+)$, and $\mathrm{P}_{\mathrm{i}}$ stands for the percentage of stained tumor cells ( $0 \%$ to $100 \%)$.

Details of other procedures are given in Supplementary materials and methods online.

\section{RESULTS}




\section{TS levels are increased in cancer cells with a mesenchymal phenotype}

We analyzed the publicly available mRNA array data obtained from the $\mathrm{NCl}-60$ panel of cancer cell lines (Stanford, Novartis and U133 databases). These cells have been previously independently determined to be of epithelial $(n=11)$ or mesenchymal $(n=37)$ nature by western blot quantification [19], and a significantly higher expression of TS (the TYMS gene) in mesenchymal-differentiated cells in all databases was found (see the data from the Stanford database in Figure 1A and from Novartis in Figure S1A). Consistently, an inverse correlation between TS and epithelial markers E-cadherin (CDH1) and micro-RNA-200c (miR-200c) and a positive correlation between TS and mesenchymal marker ZEB1 at the gene expression level in the same datasets were found, Figure S1B. The observation was also confirmed by generating a gene ratio (vimentin/E-cadherin) from the same datasets, which we validated as significantly correlating with the EMT status of the NCl-60 cells (Figure S1B and not shown). To further validate this observation at the protein level, we subjected a panel of 18 cancer cell lines (mostly belonging to the $\mathrm{NCl}-60$ panel and cultured in the same media and conditions) to a western blot analysis for TS and EMT markers, including E-cadherin, vimentin and ZEB1. As a result, a high variability in TS levels was found among the different cell lines, ranging from almost non-detectable to very high (Figure 1C). With regards to the association with the EMT status, even if some individual cases failed to fit in the TS-EMT correlation, we were able to confirm that cells with a mesenchymal-like phenotype (M-like cells, with low E-cadherin and high vimentin/ZEB1 levels) had an increased TS expression, compared to E-like cells (Figure 1D). To further confirm these data, we extracted the TS proteomic data from a publicly available online database [20] and analyzed the protein levels obtained from NCl-60 cancer cells $(n=32)$. The results again indicated an increased expression of TS in cancer cells classified as M-like, and this difference was found to be significant (Figure S1C-E). Cancer cells with an M-like phenotype are representative of tumors with an increased aggressiveness [14,21], and TS is required for the proliferation of cells $[2,8]$. In order to rule out that the observed higher TS levels in cells that have undergone EMT could have been attributable to an increased proliferation rate of the M-like cells, we analyzed the gene-array data searching for associations between well-established cancer proliferation markers (Ki67, 
cyclin-D1, PCNA and others as previously characterized [22]). However, no correlation between expression levels of other proliferation genes and EMT status of the cells was found (Figure S2A). Indeed, by the analysis of the doubling times data, we could exclude that the increased TS levels in M-like cells of the NCl-60 cells were due to an increased proliferation rate (Figure S2B). In synthesis, cancer cells with an M-like status show increased TS levels, not likely attributable to a differential proliferation rate.

\section{TS levels are associated with ZEB1}

By the analysis of both mRNA and protein levels a strong positive correlation between TS and ZEB1 was observed (quantification in Figure 1E). ZEB1 is a powerful transcriptional regulator and can serve as a master regulator of EMT in cancer cells, by directly suppressing or activating EMT-related genes [23,24]. We therefore aimed at further investigating the nature of this association. First, since the $\mathrm{NCl}-60$ group encompasses different tumor types, we aimed at determining if the TS-ZEB1 correlation was retained in cells from a single tumor type. In a panel of seven NSCLC cells, western blot analysis showed a higher TS expression in cells with increased ZEB1 levels (Figure 1F). To explore the basis for this relationship, we used a shRNAmediated knockdown approach to suppress ZEB1 in lung adenocarcinoma A549 cells, which are of an intermediate EMT status [19]. Stably infected cells showed a significant reduction of ZEB1 levels and a robust suppression of EMT, as evidenced by the reduced vimentin and the increase in E-cadherin (Figure 1G). The occurrence of MET (the opposite of EMT) was also confirmed by immunofluorescence for EMT markers (Figure S3A). Importantly, TS protein levels were also significantly reduced by ZEB1 knockdown (Figure 1G), suggesting the existence of a possible functional association between ZEB1 and TS. However, TS and vimentin mRNA levels evaluated by RT-qPCR were not found to be reduced (Figure $\mathbf{1 H}$ ), suggesting that the suppression induced by shZEB1 was not at the transcript level.

Protein lysates of cell lines derived from tumors from a genetically engineered mouse model of pancreatic cancer, Kras ${ }^{\mathrm{G} 12 \mathrm{D}} ; \operatorname{Trp} 53^{\mathrm{R} 172 \mathrm{H}} ; \mathrm{Pdx1}$-Cre $(\mathrm{KPC})$ or from the ZEB1 knockout counterpart, called KPCZ, recently generated (Krebs et al., personal communication) were additionally tested. As a result, KPCZ cells exhibited an E-like 
phenotype and significantly lower TS levels than KPC. The association was also found among KPC cells with different EMT status (Figure S3B-C). In order to test the possibility that ZEB1 can directly regulate TS expression, a doxycyclin-inducible system to overexpress ZEB1 was used in MCF-10A cells [25]. However, ZEB1 overexpression did not change TS levels in these cells (Figure S3D) and similar results were obtained transiently overexpressing ZEB1 in A549 and other cancer cells (not shown). We therefore concluded that ZEB1 regulates TS in an indirect fashion.

\section{EMT can induce TS and mesenchymal cells are more resistant to TS inhibition}

Increased TS levels are associated with resistance to TS-inhibiting agents [2,8]. To test a differential sensitivity of cells with an established EMT status, we again analyzed the datasets from the $\mathrm{NCl}-60$ panel [26]. 5-fluorouracil sensitivity data were extracted from two chemical screens and the cells divided by EMT status, according to the VIM/CDH1 gene ratio. The results indicated significantly greater resistance in cells with an $\mathrm{M}$-like phenotype (Figure 2A-B). To validate these data in our cells, we matched E-like and Mlike cells from eight cell lines of four different origins (breast, lung, colon and ovarian) and evaluated the relative 5-FU sensitivity by death assays with IC50 calculations. A lower rate of viable cells was consistently observed in E-like cells (Figure 2C-D) and this was confirmed by death assays (Figure 2E-F and Figure S4A). To confirm that EMT could be partly responsible for increased TS expression, A549 cells were subjected to EMT induction by treatment with TGF- $\beta$ (Figure $\mathbf{2 G}$ ) or by StemXVivo EMT inducing media supplement (Figure S4B), and in both cases we could detect a transitory increase in TS levels. Also, a significant increase in 5-FU resistance was found in TGF- $\beta$ treated cells (Figure 2H). To further link TS with cancer cells' EMT, we analyzed gene datasets from the GEO repository and found that TGF- $\beta$ treatment in immortalized bronchial epithelial cell (Figure S4C) and that overexpression of four independent EMT factors in human mammary epithelial cells [27] (Figure S4D) could significantly increase TS levels. Conversely, gene array data [28] from breast cancer cells with enforced overexpression of the EMT-suppressing miRNA miR-200c $[19,21,23]$ produced a significant reduction of TS mRNA expression (Figure S4E). All together 
these data support the existence of a connection between TS and EMT, with the indirect role of ZEB1.

\section{EMT-suppressing miRNAs reduce TS}

Micro-RNAs can suppress the expression of genes by targeting their 3' untranslated regions (UTR), and they have been described as strong regulators of EMT and stemlike features of cancer [12]. For a preliminary check of whether the TS 3'-UTR was targeted by miRNAs (or by other 3'-UTR-targeting elements) as previously indicated [29], we transfected a luciferase reporter plasmid containing the full-length TS 3'-UTR into A549 cells. We obtained a significant reduction of luciferase activity compared to a control plasmid (Figure 3A), suggesting a degree of targeting (not sufficient to completely abrogate TS protein expression). A similar result was also obtained by cloning the 3'-UTR sequence downstream of the stop codon of dsRed in a Redexpressing plasmid (Figure S5A). We then used a bioinformatic approach [30] to identify the miRNAs that most significantly negatively correlated with TS gene expression; the top hits were EMT-suppressing miRNAs (data on $\mathrm{NCl}-60$ cells, Figure S5B), the miR-200s, miR-203, miR-215, and miR-375 [31-34]. The last three miRNAs also displayed potential seed matches in the TYMS 3'-UTR (Figure 3B), and two of them (miR-215 and miR-203) have been already reported as targeting TS [29,35]. Further confirmation of the role of these miRNAs in EMT was found by checking the correlation scores with E-cadherin and vimentin (Figure $3 \mathbf{C}$ ). To check if these miRNAs could effectively reduce TS levels, we performed pre-miRNA transfection experiments in HCT116 and HeyA8, colon and ovarian cancer cells (intermediate-EMT and M-like status, respectively) with high TS levels, using miR-200a and miR-200c as positive control for EMT suppression. By western blotting it was observed that most of these miRNAs could significantly reduce TS levels, and that miR-375 had the strongest activity (Figure 3D-E). EMT suppression was confirmed by ZEB1 reduction and restoration of E-cadherin expression. HCT116 cells have lower miR-375 than other colon cancer cells (Figure S6A-B) and higher TS levels (Figure 1C). A luciferase reporter assay confirmed that the suppression was at the level of the 3'-UTR and the predominant role for miR-375 (Figure 3F). 


\section{ZEB1 regulates TS through miR-375}

To establish TS as a specific target of miR-375, we mutated the TS 3'-UTR in the seed match region and found that the mutated sequence could not be suppressed by miR375 (Figure 3G). Consistently, miR-375 was found to sensitize the cells to 5-FU by specific TS inhibition (Figure S6C-D). Since miR-375 has been described as negatively regulated by ZEB1 [36,37], we measured miR-375 in A549 cells with ZEB1 knockdown, and found a dramatic increase relative to control-infected cells (Figure $3 \mathbf{H}$ ). miR-203, another TS-targeting miRNA suppressed by ZEB1 [35,38], was also increased in shZEB1 cells (2.5-fold, not shown). Pre-miR-375 transfection into the parental A549 cells strongly reduced TS, while miR-375 inhibition with antagonistic miRNA in shZEB1 cells restored TS levels (Figure 3I-J). These results confirmed the specificity and the significance of miR-375 in TS regulation, indicating the indirect involvement of ZEB1.

\section{TS can promote EMT and ZEB1 expression}

To test a direct role of TS on EMT, we established a TS knockdown using two independent shRNA sequences. Western blots showed that A549 cells increased the expression of epithelial and reduced that of mesenchymal markers compared to scrambled-infected (PLKO) cells (Figure 4A). In line with the connection between EMT and CSCs, CSC markers like Oct-4 and Nanog were also reduced in shTS cells. Immunofluorescence staining confirmed the EMT suppression, with reduced vimentin and increased E-cadherin expression (Figure 4B). Since TS elimination can induce death, we verified that the observed phenomenon was not attributable solely to a differential sensitivity of E-like and M-like cells to TS suppression; a death assay by PI staining showed minimal or no death associated with TS knockdown (Figure 4C and Figure S7A). Proliferation rate, monitored by MTS and by an Incucyte real-time proliferation assay, was reduced by the shTS\#2 sequence, only, but not the shTS\#1 (not shown). Therefore, the observed effects of TS knockdown on EMT are not related to a defect in proliferation. However, a colony-formation assay indicated a reduced number of colonies also from the shTS\#1, suggesting that the cells' aggressiveness was reduced (Figure 4D). 
Along with the expression of specific markers and reduction of colony formation ability, another hallmark of EMT is the acquisition of a highly migratory phenotype. We therefore analyzed if TS suppression had an impact on cells' motility. A wound-healing assay indicated that shTS cells had impaired migration (Figure 4E-F). This was also independently confirmed by a real-time migration assay, analyzing the results with a matrix (relative wound density) that normalizes for the cells' proliferation (Figure 4G).

Another important feature of EMT is the acquisition of chemoresistance, and a real-time death assay staining cells with a green cytotoxicity reagent indicated a significant higher sensitivity of A549-shTS cells to the chemotherapeutic drug cisplatin (Figure 4H). High ALDH1 activity/expression (ALDH1 $1^{\text {high}}$ ) has been previously reported to mark a subpopulation of cells with the features of CSCs [39]. In light of the strong connection between EMT and CSCs, we also aimed at identifying a connection between TS and stemness markers. siRNA-mediated knockdown of A549 cells reduced the percentage of $A L D H 1^{\text {high }}$ cells (Figure 4I-J), and the ability of the cells to grow in sphere-forming conditions in low-attachment dishes (Figure 4K), a common readout of cancer stemness, in the absence of a dramatic reduction of the cell's ability to proliferate and grow (Figure S7B). A cell-sorting experiment confirmed increased TS levels in ALDH $1^{\text {high }}$ cells (Figure S7C). Similar results were also found in shTS cells (Figure S7D-E). As a further experiment to directly investigate the role of TS in cancer EMT and stemness, we stably overexpressed TS and we conversely found a reduction in Ecadherin and a significant increase in ZEB1 protein levels and in the relative proportion of $\mathrm{ALDH} 1^{\text {high }}$ cells (Figure $4 \mathrm{~L}-\mathbf{M}$ ), as well as an increase in sphere-forming ability (Figure S7F). Finally, in order to validate the role for TS in EMT and cancer stemness in another cellular system, we subjected a pancreatic cancer cell line from the KPC model to similar experimental approaches, and found similar results (Figure S7G-J).

\section{5-FU can induce EMT and ZEB1 in a TS-dependent manner}

Chemoresistance to TS-inhibiting drugs like 5-FU $[40,41]$ or pemetrexed $[42,43]$, has been shown to involve the EMT program, and TS levels are known to be increased 
following treatment with TS-inhibiting drugs $[8,10,43,44]$. To test a direct connection between TS levels and drug-related EMT, we established an EMT-induction model in vitro by treating the cancer cells with increasing doses of 5-FU (Figure 5A) and confirmed the occurrence of the EMT phenotype (Figure 5B-C). Western blot analysis indicated that TS levels were significantly increased in the treated cells, along with EMT markers, while this increase was attenuated in shTS cells (Figure $5 \mathrm{C}$ ). Of note, TS knockdown cells could still increase TS levels (although to a lower extent) indicating that such increase is only partially occurring at a transcriptional level, as previously described [45]. Interestingly, ZEB1 was found not regulated by the drug treatment in cells with TS stable knockdown, and Aldefluor staining (Figure 5D) and sphere formation assay (Figure 5E) confirmed for these cells that the number of CSCs was significantly reduced.

TS levels are associated with EMT markers in non-small cell lung cancer tissues and are highly prognostically relevant

To test the association between TS and ZEB1 in cancer specimens, we performed an immunohistochemical analysis of TS, ZEB1 and E-cadherin in cancerous tissues from 61 consecutive NSCLC patients (Figure S8A). Mean TS and ZEB1 levels were 50.5 (0210 ) and 18.75 (0-140, all IHC scores), respectively. Dividing the patients according to ZEB1 expression (negative tumors compared to those with IHC score $>5$ ), higher TS levels were identified in ZEB1-positive tumors (Figure 6A) consistent with the in vitro observations. Tumors with concomitant high co-expression of TS and ZEB1 were predominantly of the squamous cell carcinoma histotype $(p<0.01)$. Dividing patients according to E-cadherin negativity (either negative or cytoplasmic diffuse staining pattern) or positivity (membrane pattern of staining), as previously done [21], we also found significantly reduced TS levels in E-cadherin positive tumors (Figure 6B-C). Further dividing the patients by histology (adenocarcinoma vs. squamous-cell carcinoma), this association was significant only in patients with lung adenocarcinoma. This is probably due to the higher baseline TS in squamous-cell carcinomas [46]. No other significant association was found between the available clinical information, with the exception of a trend $(p=0.06)$ for an association with grade of differentiation and 
increased TS IHC score (Figure S8B). Analysis of survival on a large dataset of NSCLC patients [47] indicated the high prognostic relevance of TS and E-cadherin (in an opposite trend, Figure 6D) in adenocarcinoma patients, only, suggesting a biological relevance of this association in this tumor type.

\section{DISCUSSION}

Our data highlight the role of TS in cancer differentiation and EMT, showing the functional association with ZEB1 and the involvement of miRNAs, providing possible insights into the clinically observed connection between increased TS levels and local and distant metastatic spread in cancer patients [3,4,48,49].

We demonstrated significantly higher TS level in cancer cells with an M-like phenotype, not attributable to an increased propensity of these cells to proliferate. A comparative analysis of TS protein in cancer cells revealed that the expression levels could vary very significantly, and indicated that some remarkable cells have almost non-detectable expression (Figure 1C). Considering that TS is life-essential, this may indicate that minimal baseline enzyme expression is sufficient for the cells' metabolic requirements, purporting that the additional levels could be implicated in other biological functions. A significant association between TS and ZEB1, a powerful EMT-inducing transcription factor, was identified and confirmed in tumor samples. However, ZEB1 was not identified as a direct modulator of TS expression by functional assays. Searching for possible mechanisms behind the association between TS and EMT, we found that several EMT-suppressing miRNAs could reduce TS and we identified and validated miR-375 as a novel TS-targeting miRNA. The fact that multiple independent miRNAs involved in EMT can target TS further highlights the importance of its regulation in cancer differentiation. ZEB1 is known to repress EMT-suppressing miRNAs, like miR200 , miR-203 or miR-375 [24,36,37], and we found that ZEB1 knockdown up-regulated miR-375 and reduced TS expression. TS-ZEB1 co-expression may therefore be sustained by regulatory circuits involving miRNAs. Interestingly, we found that TS itself possesses EMT-regulating properties and can induce ZEB1. Since all the players 
involved in this reciprocal regulation can be simultaneously co-expressed, especially in cells with an intermediate-EMT status like A549, it is reasonable to speculate the existence of a molecular equilibrium, which can be rapidly unbalanced upon EMT induction (Figure 5F). Overall, these data represent an initial step into a better description of the previously identified role of TS in tumorigenesis [11]. Further investigations are needed to explore the possible involvement of other EMT transcription factors on TS regulation, and to determine the exact contribution of nucleotide metabolism to stemness and EMT, as previously found $[17,18]$. Finally, we confirmed recent reports showing the occurrence of EMT in cells overexpressing TS after treatment with TS inhibitors $[42,43]$, and we found that the transformation was attenuated by TS knockdown (Figure 5). These results, in the long run, may inspire the design and the clinical implementation of novel improved drugs (like small molecules inhibitors or gene-targeting agents) targeting TS without triggering its expression. With regards to possible translational implications, the EMT status of the tumors (particularly in lung adenocarcinoma) could be tested as stratification criteria for administering TSinhibiting drugs.

\section{Acknowledgments}

Work supported by the Interdisciplinary Center for Clinical Research (IZKF) at the University Hospital of the University of Erlangen-Nuremberg.

\section{Author contribution statement}

A.Siddiqui, MEV, AS, FN, CFM, performed the experiments. MPS and TB provided cells and mouse samples. IR, MV provided human paraffin-embedded materials, performed $\mathrm{IHC}$, histological evaluation and IHC scoring. MPS, TB, IR, MV and PC discussed the work. A.Siddiqui and PC designed the experiments and wrote the paper. 


\section{REFERENCES}

1. Allen TC. Targeted molecular therapy for lung cancer: exuberance reestablished. Arch Pathol Lab Med 2013; 137: 747.

2. Costi MP, Ferrari S, Venturelli A, et al. Thymidylate synthase structure, function and implication in drug discovery. Curr Med Chem 2005; 12: 2241-2258.

3. Lee HS, Chen M, Kim JH, et al. Analysis of 320 gastroenteropancreatic neuroendocrine tumors identifies TS expression as independent biomarker for survival. Int J Cancer 2014; 135: 128-37.

4. Edler D, Hallstrom M, Johnston PG, et al. Thymidylate synthase expression: an independent prognostic factor for local recurrence, distant metastasis, diseasefree and overall survival in rectal cancer. Clin Cancer Res 2000; 6: 1378-1384.

5. Saviozzi S, Ceppi P, Novello S, et al. Non-small cell lung cancer exhibits transcript overexpression of genes associated with homologous recombination and DNA replication pathways. Cancer Res 2009; 69: 3390-3396.

6. Ceppi $\mathrm{P}$, Monica V, Righi L, et al. Emerging role of thymidylate synthase for the pharmacogenomic selection of patients with thoracic cancer. Int J Clin Pharmacol Ther 2010; 48: 481-482.

7. Takezawa K, Okamoto I, Okamoto $\mathrm{W}$, et al. Thymidylate synthase as a determinant of pemetrexed sensitivity in non-small cell lung cancer. $\mathrm{Br} \mathrm{J}$ Cancer 2011; 104: 1594-1601.

8. Longley DB, Harkin DP, Johnston PG. 5-fluorouracil: mechanisms of action and clinical strategies. Nat Rev Cancer 2003; 3: 330-338.

9. Ceppi P, Papotti M, Monica V, et al. Effects of Src kinase inhibition induced by dasatinib in non-small cell lung cancer cell lines treated with cisplatin. $\mathrm{Mol}$ Cancer Ther 2009; 8: 3066-3074.

10. Ceppi P, Rapa I, Lo lacono M, et al. Expression and pharmacological inhibition of thymidylate synthase and Src kinase in nonsmall cell lung cancer. Int $J$ Cancer 2012; 130: 1777-1786.

11. Rahman L, Voeller D, Rahman M, et al. Thymidylate synthase as an oncogene: a novel role for an essential DNA synthesis enzyme. Cancer Cell 2004; 5: 341-351. 
12. Ceppi $\mathrm{P}$, Peter ME. MicroRNAs regulate both epithelial-to-mesenchymal transition and cancer stem cells. Oncogene 2014; 33: 269-278.

13. Moreno-Bueno G, Portillo F, Cano A. Transcriptional regulation of cell polarity in EMT and cancer. Oncogene 2008; 27: 6958-6969.

14. Mani SA, Guo W, Liao MJ, et al. The epithelial-mesenchymal transition generates cells with properties of stem cells. Cell 2008; 133: 704-715.

15. Magee JA, Piskounova E, Morrison SJ. Cancer stem cells: impact, heterogeneity, and uncertainty. Cancer Cell 2012; 21: 283-296.

16. Visvader JE, Lindeman GJ. Cancer stem cells: current status and evolving complexities. Cell Stem Cell 2012; 10: 717-728.

17. Bageritz J, Puccio L, Piro RM, et al. Stem cell characteristics in glioblastoma are maintained by the ecto-nucleotidase E-NPP1. Cell Death Differ 2014; 21: 929-40

18. Shaul YD, Freinkman E, Comb WC, et al. Dihydropyrimidine accumulation is required for the epithelial-mesenchymal transition. Cell 2014; 158: 1094-1109.

19. Park SM, Gaur AB, Lengyel E, et al. The miR-200 family determines the epithelial phenotype of cancer cells by targeting the E-cadherin repressors, ZEB1 and ZEB2. Genes Dev 2008; 22: 894-907.

20. Wilhelm M, Schlegl J, Hahne $\mathrm{H}$, et al. Mass-spectrometry-based draft of the human proteome. Nature 2014; 509: 582-587.

21. Ceppi P, Mudduluru G, Kumarswamy R, et al. Loss of miR-200c expression induces an aggressive, invasive, and chemoresistant phenotype in non-small cell lung cancer. Mol Cancer Res 2010; 8: 1207-1216.

22. Whitfield ML, George LK, Grant GD, et al. Common markers of proliferation. Nat Rev Cancer 2006; 6: 99-106.

23. Burk U, Schubert J, Wellner U, et al. A reciprocal repression between ZEB1 and members of the miR-200 family promotes EMT and invasion in cancer cells. EMBO Rep 2008; 9: 582-589.

24. Wellner U, Schubert J, Burk UC, et al. The EMT-activator ZEB1 promotes tumorigenicity by repressing stemness-inhibiting microRNAs. Nat Cell Biol 2009; 11: 1487-1495. 
25. Lehmann W, Mossmann D, Kleemann J, et al. ZEB1 turns into a transcriptional activator by interacting with YAP1 in aggressive cancer types. Nat Commun 2016; 7: 10498.

26. Shankavaram UT, Varma S, Kane D, et al. CellMiner: a relational database and query tool for the NCl-60 cancer cell lines. BMC Genomics 2009; 10: 277.

27. Taube JH, Herschkowitz JI, Komurov K, et al. Core epithelial-to-mesenchymal transition interactome gene-expression signature is associated with claudin-low and metaplastic breast cancer subtypes. Proc Natl Acad Sci U S A 2010; 107: 15449-15454.

28. Knezevic J, Pfefferle AD, Petrovic I, et al. Expression of miR-200c in claudin-low breast cancer alters stem cell functionality, enhances chemosensitivity and reduces metastatic potential. Oncogene 2015; 34: 5997-6006.

29. Boni V, Bitarte N, Cristobal I, et al. MiR-192/miR-215 influence 5-fluorouracil resistance through cell cycle-mediated mechanisms complementary to its posttranscriptional thymidilate synthase regulation. Mol Cancer Ther 2010; 9: 22652275.

30. Hua $Y$, Duan $S$, Murmann AE, et al. MiRConnect: identifying effector genes of miRNAs and miRNA families in cancer cells. PLoS One 2011; 6: e26521.

31. Hong $\mathrm{S}$, Noh $\mathrm{H}$, Teng $\mathrm{Y}$, et al. SHOX2 is a direct miR-375 target and a novel epithelial-to-mesenchymal transition inducer in breast cancer cells. Neoplasia 2014; 16: 279-290.

32. Khella HW, Bakhet M, Allo $G$, et al. MiR-192, miR-194 and miR-215: a convergent microRNA network suppressing tumor progression in renal cell carcinoma. Carcinogenesis 2013; 34: 2231-2239.

33. Qu Y, Li WC, Hellem MR, et al. MiR-182 and miR-203 induce mesenchymal to epithelial transition and self-sufficiency of growth signals via repressing SNAI2 in prostate cells. Int J Cancer 2013; 133: 544-555.

34. Yu X, Jiang X, Li H, et al. MiR-203 Inhibits the Proliferation and Self-Renewal of Esophageal Cancer Stem-Like Cells by Suppressing Stem Renewal Factor Bmi1. Stem Cells Dev 2014; 23: 576-585. 
35. Li T, Gao F, Zhang XP. MiR-203 enhances chemosensitivity to 5-fluorouracil by targeting thymidylate synthase in colorectal cancer. Oncol Rep 2015; 33: 607614.

36. Selth LA, Das R, Townley SL, et al. A ZEB1-miR-375-YAP1 pathway regulates epithelial plasticity in prostate cancer. Oncogene 2017; 36: 24-34

37. De Souza Rocha Simonini P, Breiling A, Gupta N, et al. Epigenetically deregulated microRNA-375 is involved in a positive feedback loop with estrogen receptor alpha in breast cancer cells. Cancer Res 2010; 70: 9175-9184.

38. Meidhof S, Brabletz S, Lehmann W, et al. ZEB1-associated drug resistance in cancer cells is reversed by the class I HDAC inhibitor mocetinostat. EMBO Mol Med 2015; 7: 831-847.

39. Kim IG, Kim SY, Choi SI, et al. Fibulin-3-mediated inhibition of epithelial-tomesenchymal transition and self-renewal of $\mathrm{ALDH}+$ lung cancer stem cells through IGF1R signaling. Oncogene 2014; 33: 3908-3917.

40. Harada K, Ferdous T, Ueyama Y. Establishment of 5-fluorouracil-resistant oral squamous cell carcinoma cell lines with epithelial to mesenchymal transition changes. Int J Oncol 2014; 44: 1302-1308.

41. Zhang W, Feng M, Zheng G, et al. Chemoresistance to 5-fluorouracil induces epithelial-mesenchymal transition via up-regulation of Snail in MCF7 human breast cancer cells. Biochem Biophys Res Commun 2012; 417: 679-685.

42. Liang SQ, Marti TM, Dorn $\mathrm{P}$, et al. Blocking the epithelial-to-mesenchymal transition pathway abrogates resistance to anti-folate chemotherapy in lung cancer. Cell Death Dis 2015; 6: e1824.

43. Chiu LY, Hsin IL, Yang TY, et al. The ERK-ZEB1 pathway mediates epithelialmesenchymal transition in pemetrexed resistant lung cancer cells with suppression by vinca alkaloids. Oncogene 2017; 36: 242-253.

44. Giovannetti E, Lemos C, Tekle C, et al. Molecular mechanisms underlying the synergistic interaction of erlotinib, an epidermal growth factor receptor tyrosine kinase inhibitor, with the multitargeted antifolate pemetrexed in non-small-cell lung cancer cells. Mol Pharmacol 2008; 73: 1290-1300. 
45. Liu J, Schmitz JC, Lin X, et al. Thymidylate synthase as a translational regulator of cellular gene expression. Biochim Biophys Acta 2002; 1587: 174-182.

46. Ceppi P, Volante M, Saviozzi $S$, et al. Squamous cell carcinoma of the lung compared with other histotypes shows higher messenger RNA and protein levels for thymidylate synthase. Cancer 2006; 107: 1589-1596.

47. Gyorffy B, Surowiak P, Budczies J, et al. Online survival analysis software to assess the prognostic value of biomarkers using transcriptomic data in nonsmall-cell lung cancer. PLoS One 2013; 8: e82241.

48. Hashimoto $\mathrm{H}$, Ozeki $\mathrm{Y}$, Sato $\mathrm{M}$, et al. Significance of thymidylate synthase gene expression level in patients with adenocarcinoma of the lung. Cancer 2006; 106: 1595-1601.

49. Lee SW, Chen TJ, Lin LC, et al. Overexpression of thymidylate synthetase confers an independent prognostic indicator in nasopharyngeal carcinoma. Exp Mol Pathol 2013; 95: 83-90. 


\section{FIGURE LEGENDS}

Figure 1. TS levels are increased in cells with a mesenchymal-like phenotype. (A) Logtransformed TS (TYMS) mRNA expression levels in epithelial (green) and mesenchymal-like (red) cancer cell lines from the NCl-60 Stanford array dataset. Lines indicate median levels in both groups. (B) Correlation between E-cadherin ( $C D H 1)$, ZEB1 and miR-200c and the TYMS gene. R-values are Pearson coefficients. (C) Western blot analysis of TS protein levels and EMT markers in cancer cells. $\beta$-actin is used as a loading control. (D) Densitometric quantification (ImageJ) of TS levels expressed as ratios with $\beta$-actin in the western blots as in (C). (E) Quantification of TS levels expressed as band area intensity in the western blots as in (C), dividing for ZEB1 negativity or positivity. (F) Western blot analysis of TS and ZEB1 in a panel of non-small cell lung cancer (NSCLC) cell lines. (G) Western blot analysis of TS and EMT markers in A549 cells stably overexpressing a scrambled control or a shRNA targeting ZEB1. (H) qPCR analysis of EMT markers and TS in shZEB1 and control A549 cells. Data are $\pm S D$, normalized to GAPDH and expressed as -fold-change relative to control cells. Pvalues are two-tailed $t$-tests; ${ }^{* *}$ indicates $\mathrm{p}<0.01$.

Figure 2. A mesenchymal-like phenotype and higher TS levels are associated with

increased 5-FU resistance. (A) Sensitivity of $\mathrm{NCl}-60$ cancer cell lines to 5-FU, expressed as log-transformed values. Cells were divided into Epithelial, Mesenchymal Low and Mesenchymal High, according to a pre-determined EMT gene ratio. Data are from DTP of the National Cancer Institute $(\mathrm{NCl})$. (B) $I_{50}$ values of $\mathrm{NCl}-60$ cancer cell lines relative to 5-FU. Data are from the $\mathrm{Gl} 50$ database $(\mathrm{NCl})$. (C) Representative images of breast (MDAMB231, T47D), colorectal (HCT116, KM12), lung (A549, Calu-1) and ovarian (Ovcar5, Ovcar8) cancer cells treated with the indicated concentrations of the drug. (D) Dose-response curves and $I C_{50}$ calculations of cells as in $(C)$ treated for 48 hours with the indicated concentrations of 5 -FU. Numbers indicate $I C_{50}$ values $\pm S D$. Percentage of dead cells were calculated with FACS dead/live cells gating in quadruplicate conditions. (E) FACS plots of Ovcar8 and Ovcar5 cells treated with $1 \mathrm{mM}$ 5-FU and stained after $48 \mathrm{~h}$ with $\mathrm{PI}$ (Nicoletti). Indicated are percentages subG1 fraction 
(gated). (F) Quantification of percentage of subG1 fraction, in cells treated as in (E). (G) Western blot of E-cadherin, ZEB1 and TS in A549 cells treated for $48 \mathrm{~h}$ with $10 \mathrm{ng} / \mu \mathrm{l}$ TGF- $\beta$. (H) Percentage of viable cells in A549 cells treated and untreated with $10 \mathrm{ng} / \mu \mathrm{l}$ TGF- $\beta$ and with 5 -FU or control for the indicated time-points. All non-treated controls were given DMSO concentrations equivalent to the highest 5-FU administered dose. $\mathrm{NT}=$ non-treated and P-values are two-tailed $t$-tests, ${ }^{*}$ indicates $\mathrm{p}<0.05$.

Figure 3. EMT-suppressing miRNAs can inhibit TS expression. (A) Relative luciferase activity of A549 cells transiently transfected with Psicheck2 plasmid carrying the fulllength TS 3'-UTR or the control empty plasmid. (B) Sequence of the TS 3'-UTR starting at the stop codon. Indicated are the seed regions complementary to the indicated miRNAs: miR-215, miR-203 and miR-375. (C) Correlation scores of miR-203, miR-215 and miR-375 with the mRNA expression levels of E-cadherin ( $C D H 1$ gene) and vimentin (VIM). Data from CellMiner.com and correlation scores are according to Pearson. (D) Western blot analysis of TS, ZEB1 and E-Cadherin in HCT116 and (E) HeyA8 cells $72 \mathrm{~h}$ after transfection with the pre-miR-control, pre-miR-200a, pre-miR-200c, pre-miR-203, pre-miR-215 and pre-miR-375 at $50 \mathrm{nM}$ concentration. (F) Relative luciferase activity of 293T cells transiently transfected with the Psicheck2 plasmid carrying the full-length TS 3'-UTR, and co-transfected with pre-miR-control, pre-miR-200a, pre-miR-200c, pre-miR203, pre-miR-215 and pre-miR-375 at $50 \mathrm{nM}$ concentration, $48 \mathrm{~h}$ after transfection. (G) Relative luciferase activity of $293 \mathrm{~T}$ cells transiently transfected with the Psicheck2 plasmid carrying the full-length TS 3'UTR or with the sequence mutated in the miR-375 seed region, co-transfected with pre-miR-control or pre-miR-375. (H) qPCR quantification of miR-375 levels in A549 cells stably overexpressing an shRNA targeting ZEB1, relative to the control cells. (I) Western blot quantification of TS and ZEB1 levels in A549 cells after pre-miR-375 transfection, compared to control-transfected cells. $(\mathrm{J})$ Western blot quantification of TS and ZEB1 levels in shZEB1-A549 cells after anti-miR375 transfection, compared to control-transfected cells. P-values are two-tailed $t$-tests ( ${ }^{*}$ indicates $p<0.05,{ }^{* *}$ indicates $p<0.01$ ). 
Figure 4. TS suppression by shRNA reduces EMT and the migratory phenotype. (A) Western blot analysis of TS and indicated EMT and CSCs markers in A549 cells infected with shRNA targeting two independent TS mRNA sequences, compared to scrambled-infected cells (PLKO). (B) Immunofluorescence on A549 cells stably overexpressing a scrambled control or a shRNA targeting TS, stained for E-cadherin and vimentin. The cells were stained concomitantly with the shZEB1 shown in the Figure S3A, with the same PLKO cells as control. DAPI is used as a nuclear counterstain. (C) FACS plots of cells as in (A) 6 days after infection stained with PI (Nicoletti). Indicated are percentages of subG1 nuclei. (D) Colony formation of cells as in (A) stained after $7 \mathrm{~d}$ with crystal violet and quantified in triplicate dishes. (E) Representative pictures and $(F)$ quantification of wound-healing assay on A549 cells infected with non-targeting or with shTS overexpressing viruses. (G) Plots of relative wound densities of A549 cells infected with non-targeting or with shTS and shZEB1 overexpressing viruses. $(\mathrm{H})$ Real-time analysis of cell death in A549 cells infected with non-targeting or with shTS and shZEB1 overexpressing viruses after treatment with Cisplatin (CDDP) at $750 \mu \mathrm{M}$ dose. Cell death was monitored with the incorporation of a green dye by Incucyte ZOOM system. (I) Western blot analysis on TS in A549 cells transfected with siRNA smart pool targeting TS, compared to non-targeting control cells. (J) FACS plots of cells as in (I) and stained with Aldefluor reagent, to quantify the percentage of CSCs cells. Gates were set using DEAB reagent; percentages indicate the proportion of ALDH1 $1{ }^{\text {high }}$ cells. (K) Quantification of sphere number in cells treated as in (I) and plated in sphere-forming conditions in low-adherence plates. (L) Western blot analysis on TS, ZEB1 and E-Cadherin in A549 cells infected with a vector overexpressing TS, compared to control-infected cells. (M) FACS plots of cells as in (L) and stained with Aldefluor reagent. P-values are two-tailed $t$-tests ( ${ }^{*}$ indicates $p<0.05$, ${ }^{* *}$ indicates $p<0.01)$.

Figure 5. 5-FU treatment induces EMT and increases TS levels, which are required for ZEB1 activation. (A) Scheme of the EMT induction via long-term 5-FU treatment in A549 cells. Pictures show the shape of the cells treated with increasing concentration of 5-FU, up to $10 \mu \mathrm{M}$, and cells treated with DMSO (NT) after one week of growth in 
normal media. (B) Immunofluorescence on cells treated as in (A), stained for E-cadherin and vimentin. DAPI is used as a nuclear counterstain. (C) Western blot analysis on PLKO and shTS A549 cells treated as indicated in (A) and analyzed for the protein levels of EMT and CSCs markers. (D) FACS plots of cells as in (C) and stained with Aldefluor reagent, to quantify the percentage of CSCs cells. (E) Quantification of spheres number in cells treated as in (C) and plated in sphere-forming conditions in lowadherence plates. P-values are two-tailed $t$-tests $\left({ }^{*}\right.$ indicates $\left.p<0.05\right) .(F)$ Scheme of the proposed model behind TS and ZEB1 association in cancer cells, in which TS, ZEB1 and miRNAs belong to an EMT regulatory loop. EMT-suppressing miRNAs can target TS and ZEB1. Upon EMT, induced for instance by 5-FU treatment, TS and ZEB1 levels are increased and EMT-suppressing miRNAs are object of ZEB1 inhibition.

Figure 6. TS protein levels are associated with ZEB1 and E-Cadherin in NSCLC tissues. (A) Quantification of TS expression levels in tissues from 61 NSCLC patients. Patients were divided into two groups according to ZEB1 IHC positivity or negativity. (B) Quantification of TS expression levels in tissues from NSCLC patients. Patients were divided into two groups according to E-cadherin IHC negativity (either negative or cytoplasmic diffuse staining pattern) or positivity (membrane pattern of staining). Patients were further divided in adenocarcinoma (ADC) and squamous cell-carcinoma (SSC). The p-values are from a Mann-Whitney $U$ test. (C) Representative microscopic images of a NSCLC case with high (\#1) and low (\#2) TS staining, with corresponding staining of E-cadherin and ZEB1. D) Survival curves of NSCLC patients divided by high and low expression of TS (TYMS gene) and E-cadherin (CDH1 gene) from a transcriptomic database and divided for histological diagnosis (ADC and SSC). P-values indicated are log-rank, and ns=non-significant. 


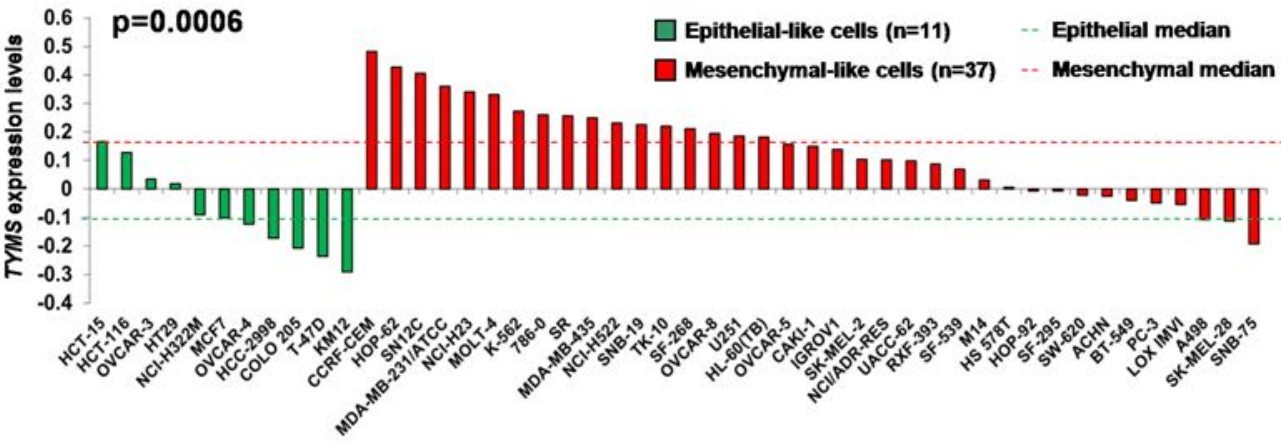

B

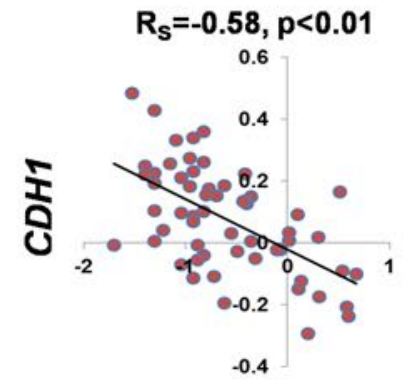

TYMS

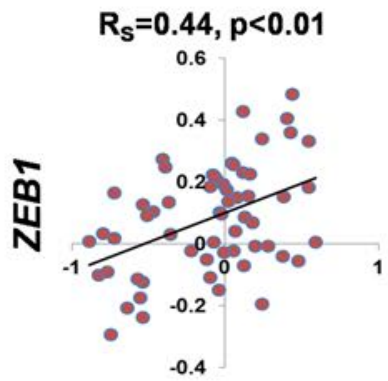

TYMS

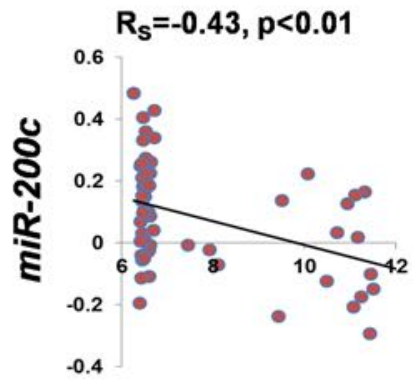

TYMS


ZEB1 neg pos

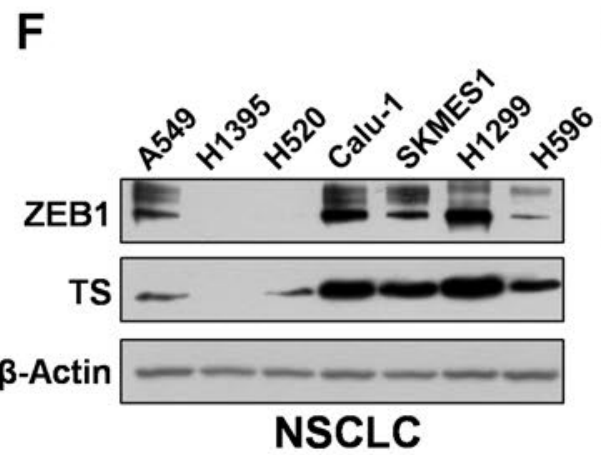

G



A549

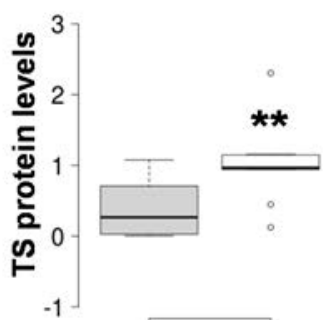

Epith. Mesench.

$\mathrm{H}$

Fold change

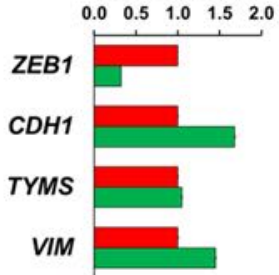

- PLKO = shZEB1 
A

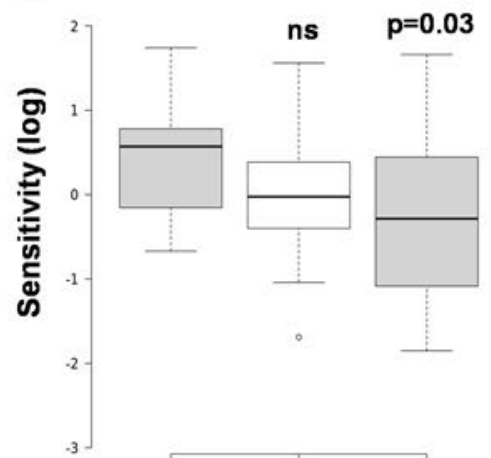

E M Low M High

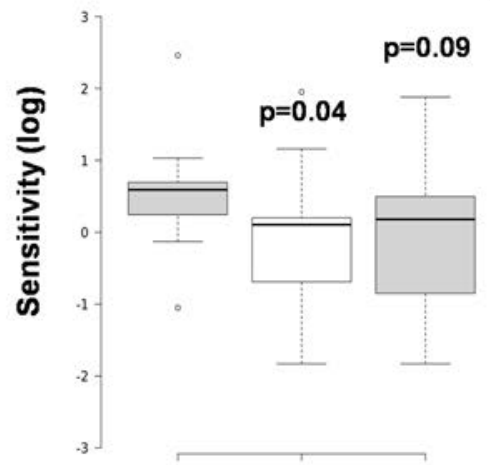

E M Low M High
B

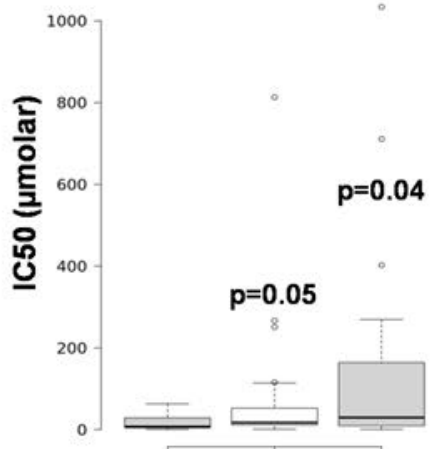

E M Low M High

C



D

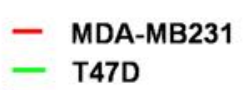

- НCT116

- Calu-1

- Ovcar8

- T47D

- KM12

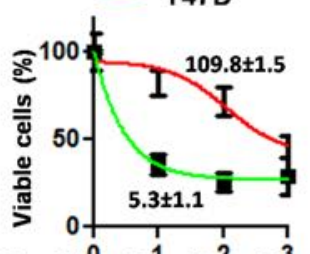

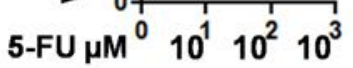

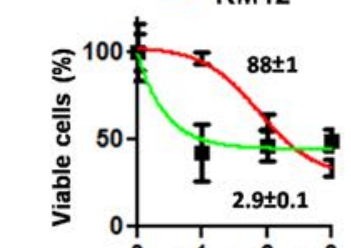

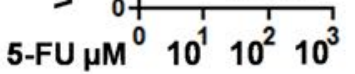

- A549

- Ovcar5
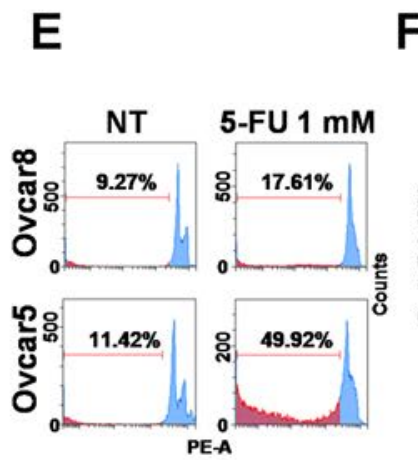

$\mathbf{F}$

G

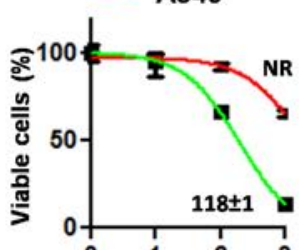

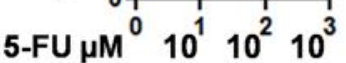



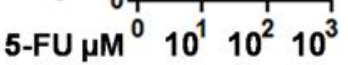
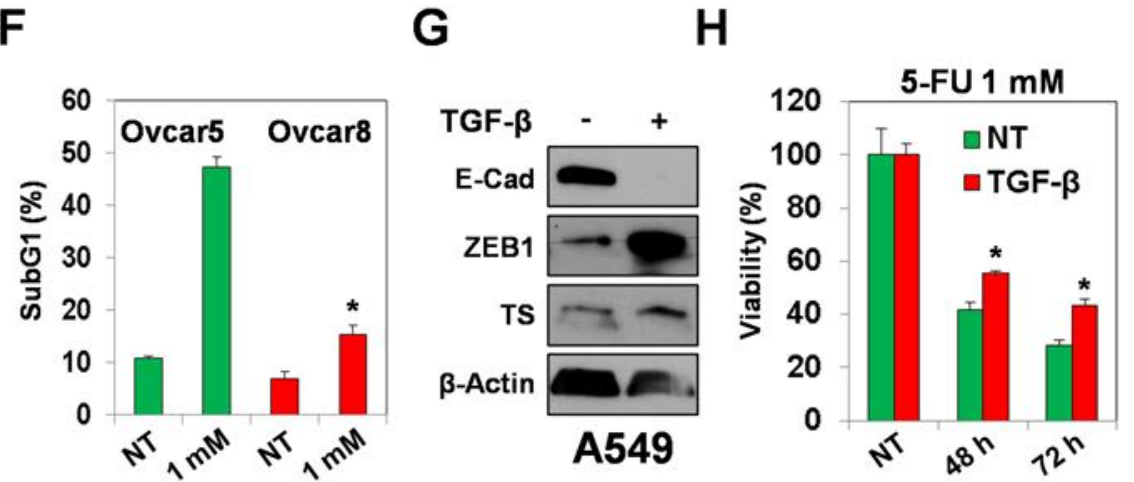
A



B

TYMS 3'UTR (502bp)

TAGGGTGCTITCAAAGGAGCTCGAAGGATATTGTCAGTCTIT AGGGGTTGGGCTGGATGCCGAGGTAAAAGTTCTITTTGCTC

TAAAAGAAAAAGGAACTAGETCIAAAATCTGTCCGTGACCT ATCAGTTATTAAITITTAAGGATGTTGCCACTGGCAAATGTA ACTGTGCCAGTTCTITCCATAATAAAAGGCTITGAGTTAACT CACTGAGGGTATCTGACAATGCTGAGGTTATGAACAMAGTG AGGAGAATGAAATGTATGTGCTCTTAGCAAAAACATGTATGT GCATTTCMTCCCACGTACTTATAAAGAAGGITGGTGAAITT CACAAGCTATITITGGAATATIITTAGAATATITTAAGAATT CACAAGCTATTCCCTCAAATCTGAGGGAGCTGAGTAACACC ATCGATCATGATGTAGAGTGTGGTTATGAACTTTAAAGTTAT AGTTGTITTATATGTTGCTATAATAAAGAAGTGTTCTGCATTC GTCAAAAAAAAAAAAAAAAAAAA

Stop codon

miR-215 CAGACAGUUAAGU UUCCDGEIA

miR-203 GAUCACCAGGAUUUGUAMGUG miR-375 AGUGCGCUCGGCUUGCUUGUUU
C

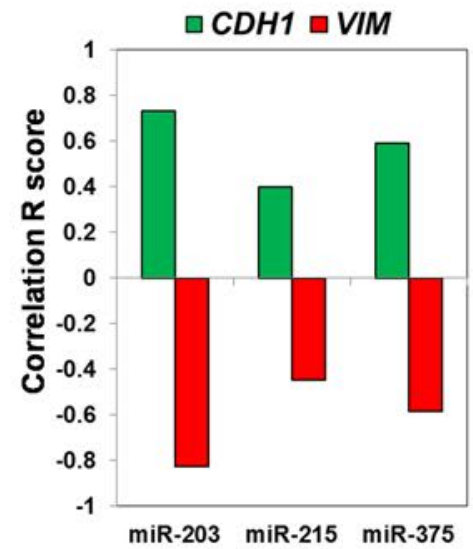

D

Pre-miR


HCT116

F

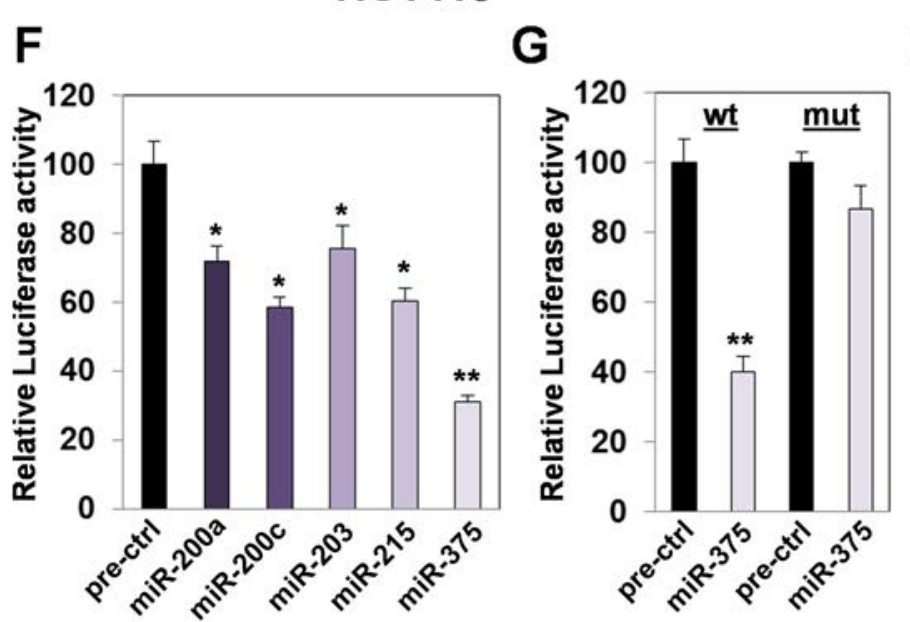

E

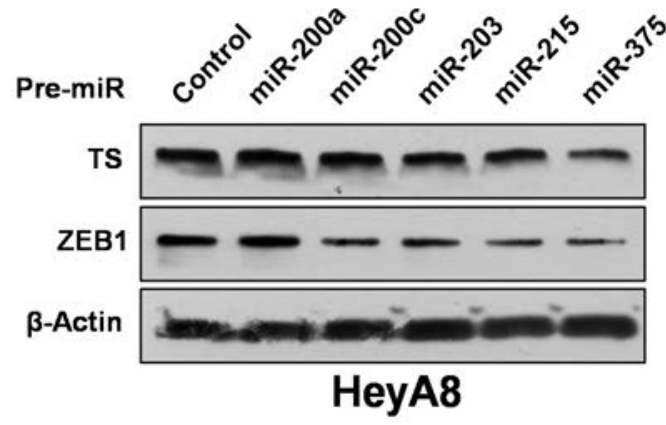

H

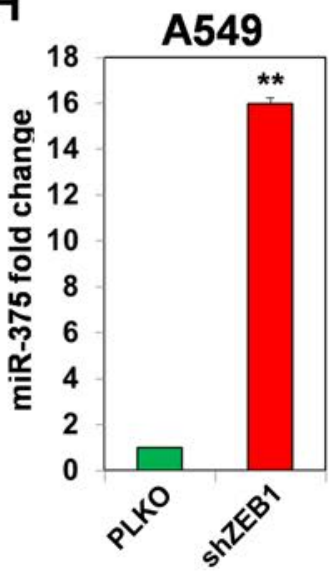

I 
A

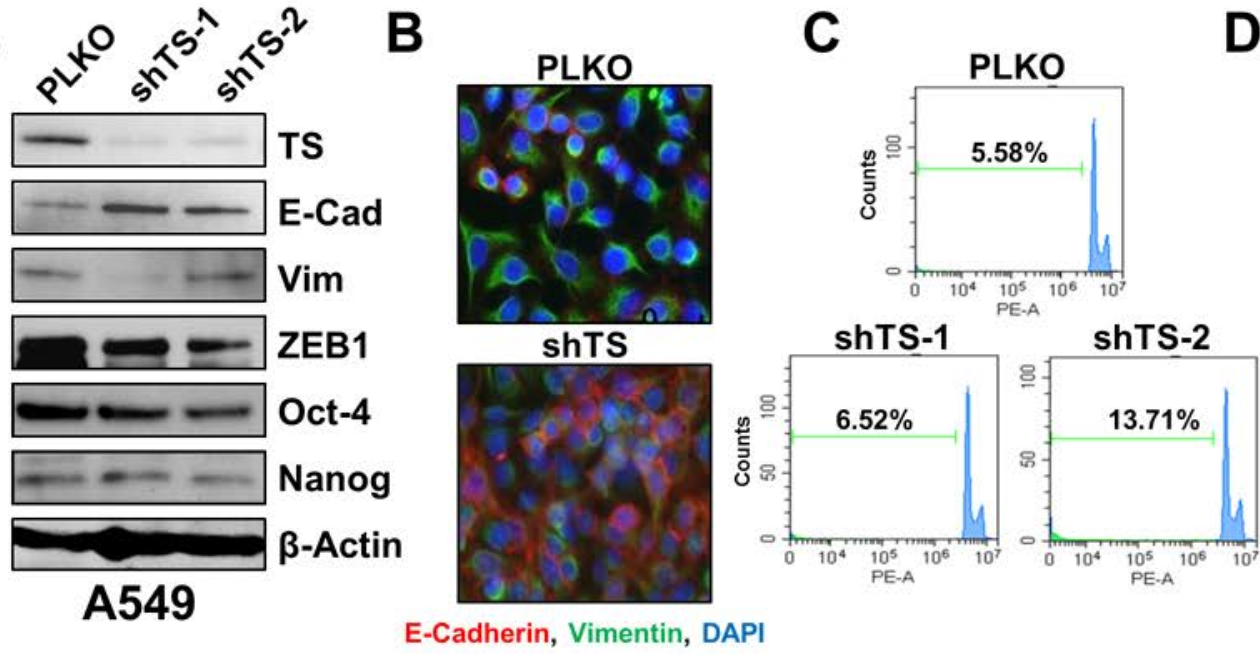

E

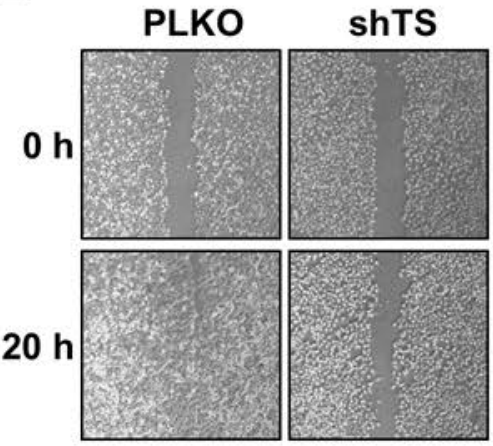

H


G

J
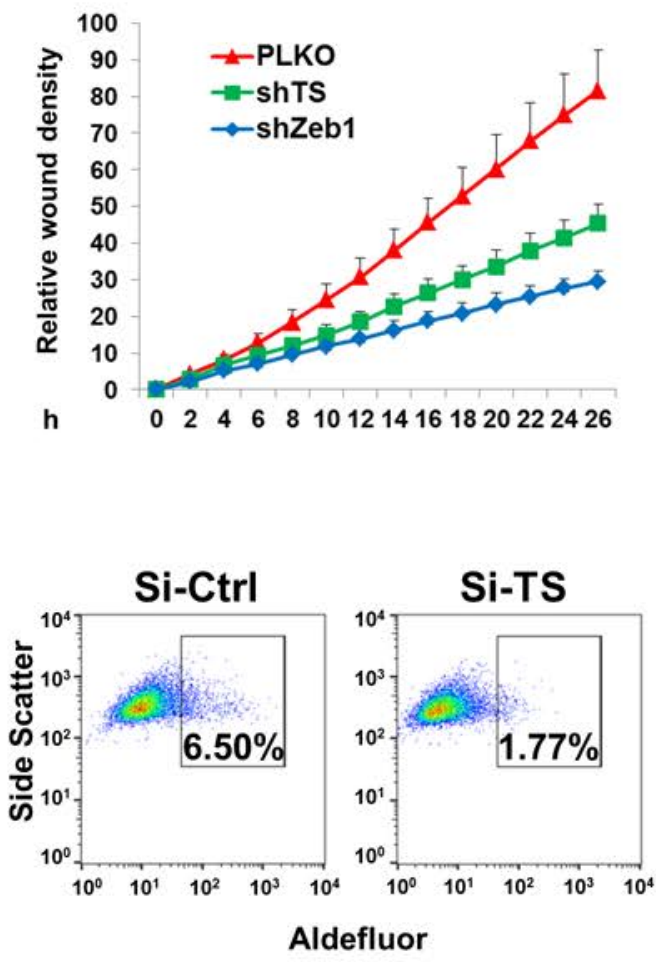

M

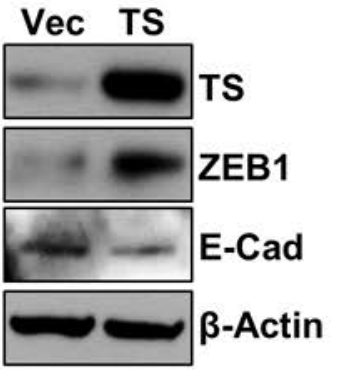

A549



Aldefluor 
A
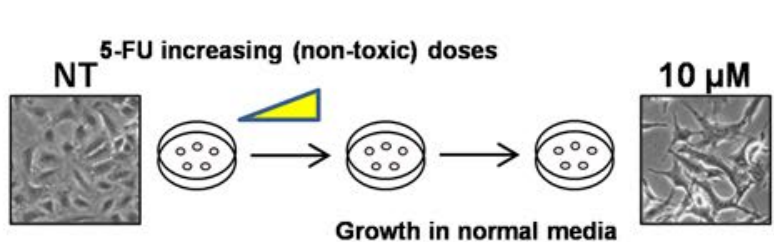

B

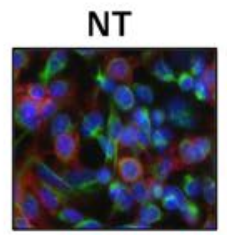

$10 \mu \mathrm{M}$ 5-FU

E-Cadherin, Vimentin, DAPI

C PLKO shTS

5-FU NT $10 \mu \mathrm{M}$ NT $10 \mu \mathrm{M}$



A549
D
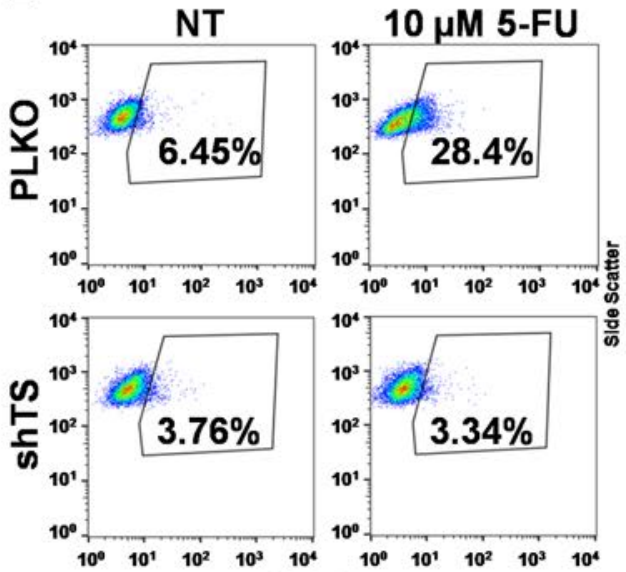

ZEB1

Oct-4

$\beta$-Actin

Aldefluor

E

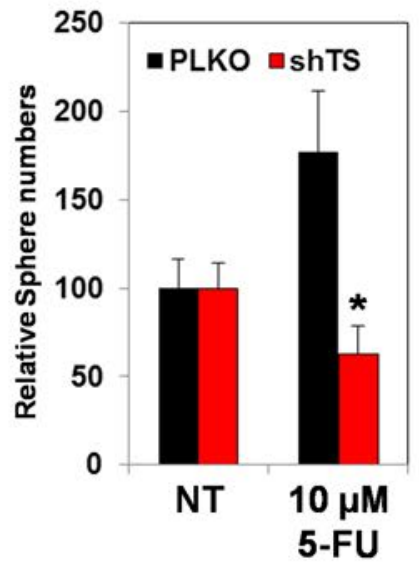

$\mathbf{F}$

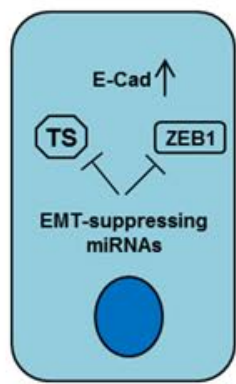

Epithelial-like Well-differentiated Low-TS

Chemosensitive

Non-CSCs
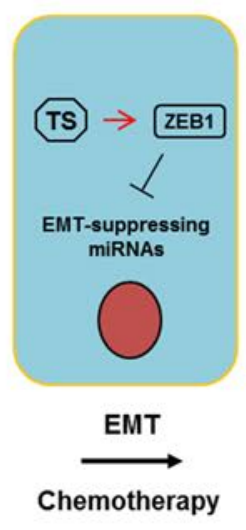

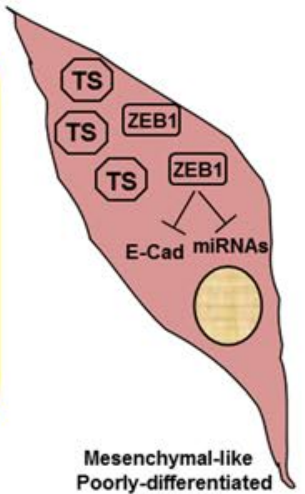

High-TS

Chemoresistant

More-CSCs 
A

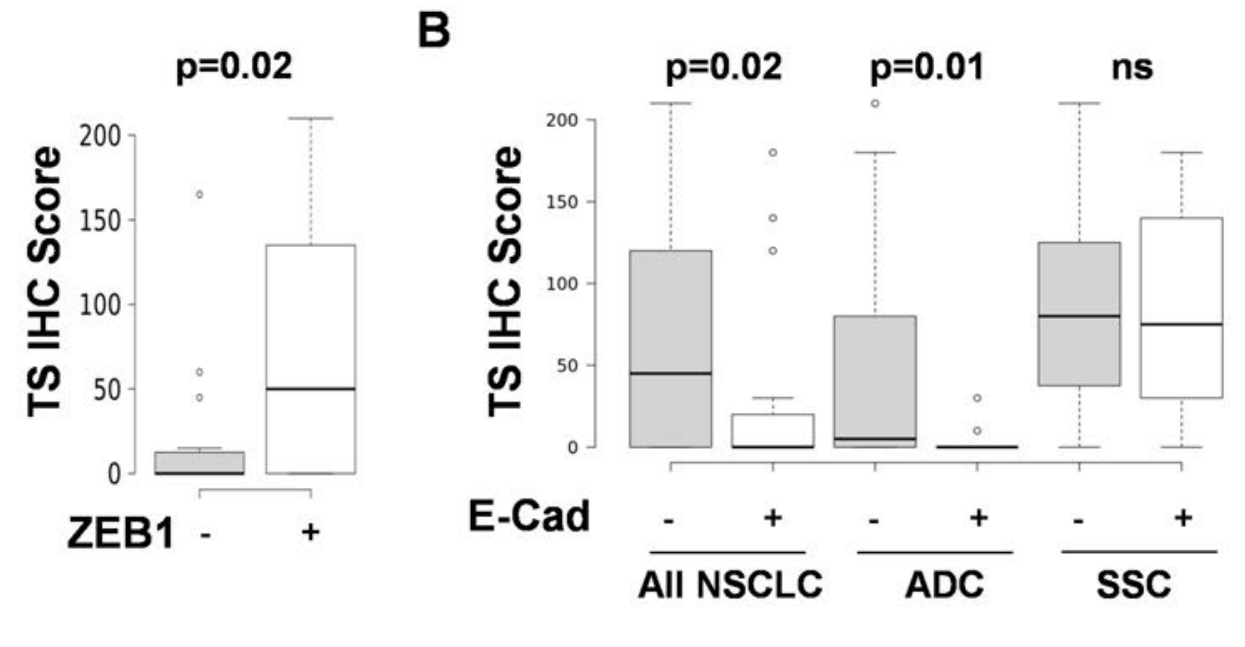

C
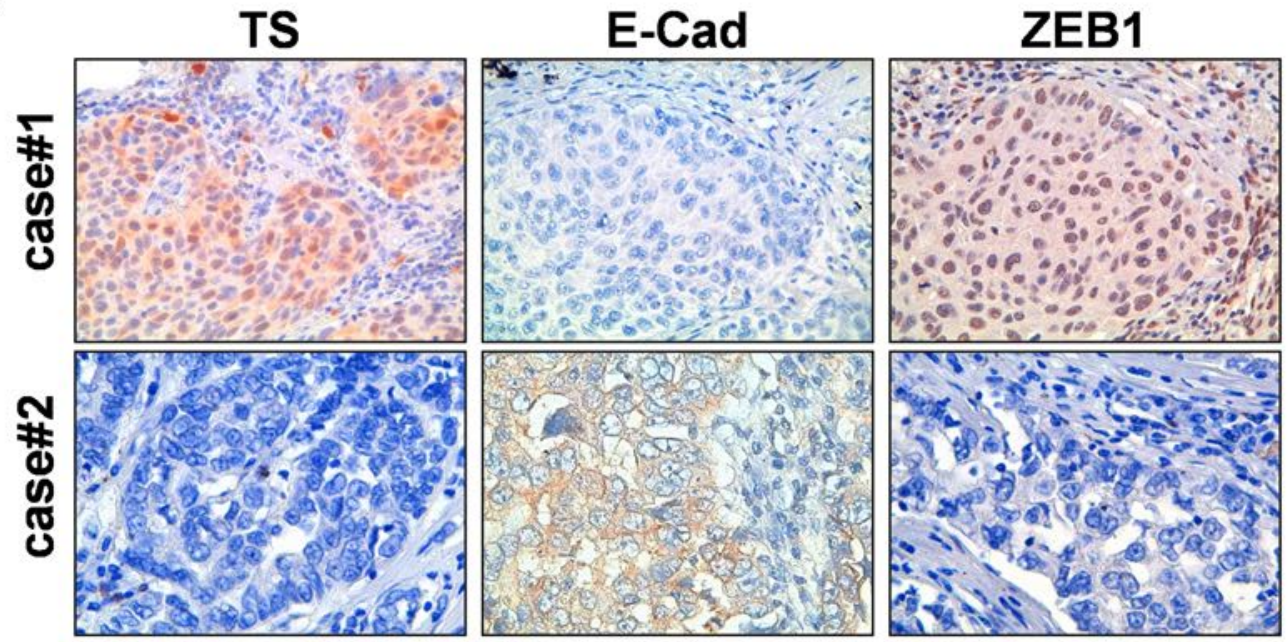

D
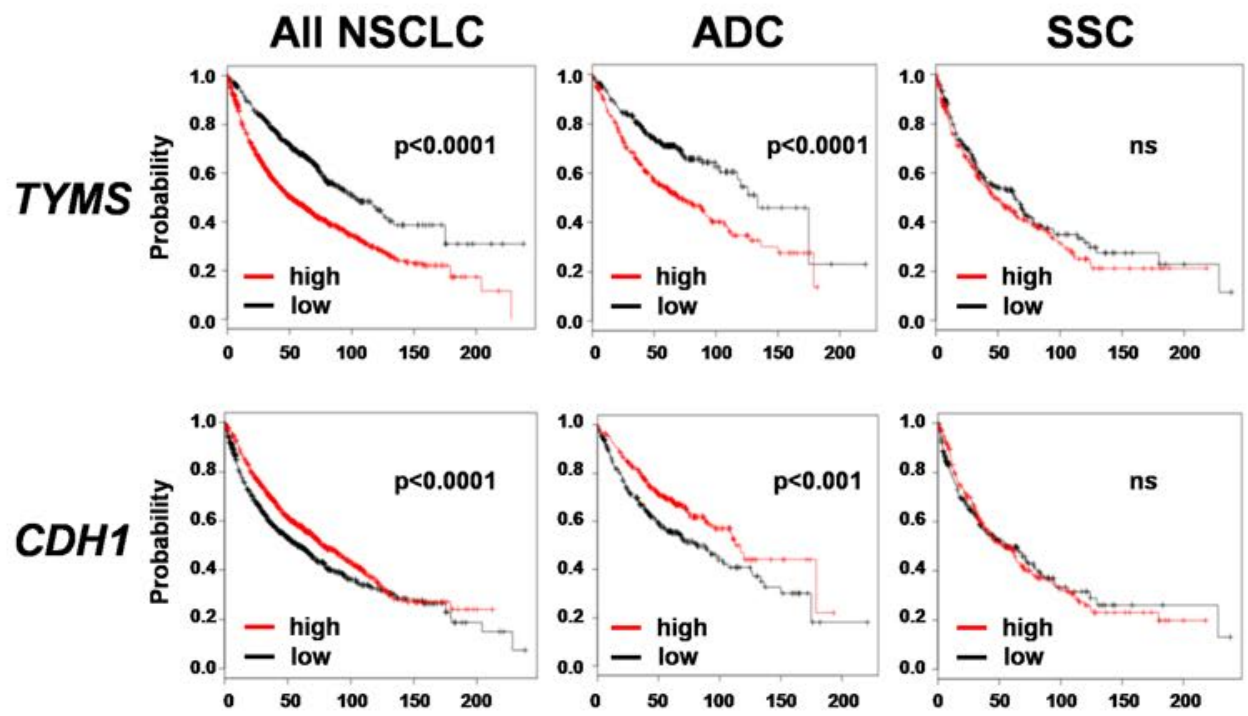